\title{
Application of synthetic peptides in development of a serologic method for laboratory diagnosis of schistosomiasis mansoni
}

\author{
Edward José de Oliveira/ ${ }^{+}$, Hermínia Yohko Kanamura/*, Kioko Takei, \\ Rosario Dominguez Crespo Hirata, Nga Yen Nguyen**, Mário Hiroyuki Hirata
}

Laboratório de Imunologia Clínica, Faculdade de Ciências Farmacêuticas, Universidade de São Paulo, Av. Prof. Lineu Prestes 580 , Bloco 17, 05508-900 São Paulo, SP, Brasil *Departamento de Biologia, Universidade de Taubaté, Taubaté, SP, Brasil

**Center for Biologics Evaluation and Research, Food and Drug Administration, Bethesda, MD, US

The immunoreactivity of seven peptides synthesized from Schistosoma mansoni proteins, was evaluated by dotblot and ELISA assays using two different sensitization methodologies. The best results were obtained on wells of the Costar 3590 microplates coated with peptides P1, P2, P3, P6, and P7 using conventional methodology. The signals increased considerably $(p<0.0003)$ on wells sensitized with P1 to P6 using alternative methodology. In contrast, the well coated with peptide P7 presented lower signal when compared with conventional methodology $(p=$ 0.0019). These results, establish the basis for the application of synthetic peptides for laboratory diagnosis of schistosomiasis mansoni.

Key words: Schistosoma mansoni - immunodiagnosis - synthetic peptides

Numerous antigens and laboratory test systems have been described for the detection of Schistosoma mansoni infections in humans. Different antigenic preparations that are considered useful for diagnosis include worm proteins Sm31 and Sm32 (El-Sayed et al. 1998), gut-associated polysaccharide (Oliveira et al. 2005), and heat-shock protein 70 (Kanamura et al. 2002).

Cathepsin B (31 kDa) and asparaginil endopeptidase $(32 \mathrm{kDa})$ are enzymes involved with the degradation of hemoglobin that is swallowed by adult worm of S. mansoni (Tort et al. 1999). These enzymes were shown to be highly immunogenic in mice and humans and they were proposed as potential serodiagnostic antigens for detection of $S$. mansoni (Klinkert et al. 1991, El-Sayed et al. 1998).

Gut-associated antigens, such as circulating anodic antigen (CAA) and circulating cathodic antigen (CCA) are glycoproteins extensively studied as targets for immunodiagnosis of schistosomiasis (Deelder et al. 1980). Both antigens were demonstrated in the serum and urine of hosts infected with $S$. mansoni (Deelder et al. 1978). Despite of these evidences, the roles of CAA and CCA in the biology of schistosomes are not clear. It has been suggested that CAA may serve as a lining to protect the schistosome gut against harmful host components. On the other hand, it may also have immunomodulatory functions by interaction with the first complement component C1q of the host (Van Dam et al. 1993).

Financial support: $\mathrm{CNPq}$

This work was performed in collaboration with the Center for Biologics Evaluation and Research, FDA, Bethesda, MD, US

${ }^{+}$Corresponding author: edwardjo@usp.br

Received 25 May 2006

Accepted 26 June 2006
The heat shock proteins 70 (HSP70) is one of the most abundant family of heat shock proteins expressed by all living organisms, both eukaryotic and prokaryotic (Moseley 2000). The use of recombinant S. mansoni HSP70 (SmHSP70) for immunodiagnostic purposes was suggested (Moser et al. 1990), but few reports are found in the literature about the frequency of anti-SmHSP70 antibodies in healthy individuals and in patients with autoimmune diseases (Arora et al. 1995). There is also a need to better identify the epitopes of the HSP70 specifically recognized by the host (Kanamura et al. 2002).

In previous work we selected five clones of an adult $S$. mansoni cDNA library designated as ET01, ET03, ET04, ET06, ET10 (Valli 2001). According to the sequence analysis of these clones, two were identified as homologous to HSP70, one to glutathione-S-transferase (GST), one to homeodomain protein, and one to a previously described EST (expressed sequence tag) of $S$. mansoni, called MA002456.C8F (GenBank, Accession no. AI395117), obtained by Bailey et al. (no published data). This last clone (ET03) was the most consistently reactive during the screening process with the anti-Sm31/32 antibodies, eluted from nitrocellulose strips after dissociation of the immune complexes. The translation data of the DNA insert of clone ET03 yielded a small open reading frame (ORF) that codes for a hydrophobic protein with 57 amino acids.

Seven peptides (P1 to P7), with 20 amino acids each were selected, based on sequences deposited in GenBank (http:/www.ncbi.nlm.nih.gov/Genbank/index.html), related to the following $S$. mansoni antigenic proteins: $\mathrm{Sm} 31$ fraction (cathepsin B) (GenBank accession no. M21309), SmHSP70 (GenBank accession no. L02415), and CCA (GenBank accession no. O02197). It also included the sequence of the ORF phase of the clone ET03, obtained according to Valli (2001). These peptides were selected after analysis of the flexibility indices and the hydrophilicity algorithms (Hopp \& Woods 1983), using the data and tools available in the site http://au.expasy.org/cgibin/protscale.pl (Table). 
TABLE

Sequences of the synthetic peptides selected from Schistosoma mansoni proteins and their corresponding designation

\begin{tabular}{|c|c|c|c|c|c|}
\hline Identification & Amino acid sequence & Original protein & $\mathrm{AA}^{b}$ & $\mathrm{Ip}^{c}$ & $\mathrm{MW}^{d}$ \\
\hline $\mathrm{P} 1^{a}$ & DHNDWNVEIPSNFDSRKKWP & Cathepsin B (Sm31) & $81-100$ & 5.43 & 2.485 \\
\hline $\mathrm{P} 2^{a}$ & QRKYKTPYTQDKHRGKSSYN & Cathepsin B (Sm31) & $221-240$ & 10.12 & 2.486 \\
\hline $\mathrm{P} 3^{a}$ & VKNDEKAIQKEIMKYGPVEA & Cathepsin B (Sm31) & $241-260$ & 6.22 & 2.291 \\
\hline P4 & KGEKKMFSAEEISSMVLTKM & HSP-Sm70 & $106-125$ & 8.43 & 2.275 \\
\hline P5 & ENKQLEQLKIENKTLRNSLD & CCA & $81-100$ & 6.41 & 2.414 \\
\hline P6 & HRQFCETKLIVLCRIFSNTH & ET03 clone & $5-24$ & 8.96 & 2.446 \\
\hline P7 & TVESLAIIILLKCAQVCIKI & ET03 clone & $34-53$ & 7.72 & 2.172 \\
\hline
\end{tabular}

$a$ : amino acid sequence based on Noya et al. (2001); $b$ : amino acid position in the sequence of the original protein; $c$ : isoelectric point; $d$ : molecular weight.

The peptides were synthesized by Fmoc solid phase peptide synthesis at the Center for Biologics Evaluation and Research at Food and Drug Administration, Bethesda, MD, US.

The immunoreactivity of the peptides was evaluated primarily by dot-blot assays using nitrocellulose and PVDF membrane against pools of serum samples from individuals who tested positive and negative for schistosomiaisis. The peptides P1 to P7 were immobilized on nitrocellulose or PVDF membrane from different origins. Membranes were exposed to a pool of serum samples collected from five schistosomiasis patients, confirmed by parasitologic and serologic methods. The negative control consists of a pool of serum samples obtained from five individuals with negative results for parasitologic and serologic tests. A biotin-conjugated anti-human IgG antibodies and an avidin-conjugated peroxidase were used, and the reaction was revealed with a solution of 4-chloro$\alpha$-naphthol and hydrogen peroxide. However, only peptide $\mathrm{P} 7$ presented immunoreactivity against positive sera pool using PVDF membrane. Apparently the other peptides have not fixed on nitrocellulose or PVDF membrane (results not shown).

We also tested the peptides by ELISA. Briefly, 96-well flat-bottom microtiter plates were sensitized by incubation overnight at $4^{\circ} \mathrm{C}$ with peptides 1 to $7(50 \mu \mathrm{l}$ per well $)$ diluted in $0.1 \mathrm{M}$ carbonate/bicarbonate buffer, $\mathrm{pH} 9.6$ (conventional methodology). Plates were washed five times with PBS containing $0.05 \%$ Tween 20 (PBS-Tween) and blocked with PBS-Tween containing 5\% skim milk (PBSTween-5\% milk) for $2 \mathrm{~h}$ at $37^{\circ} \mathrm{C}$. After washing, $50 \mu \mathrm{l} \mathrm{per}$ well of serum pools diluted to 1:20 with PBS-Tween-1\% milk were added to the wells and incubated for $30 \mathrm{~min}$ at $37^{\circ} \mathrm{C}$. A peroxidase-conjugated anti-human IgG (Sigma Chemical Company, St. Louis, MO, US) diluted to 1:1000 in PBS-Tween-1\% milk was added to each wells and incubated for $30 \mathrm{~min}$ at $37^{\circ} \mathrm{C}$. Hydrogen peroxide (Merck S.A., Rio de Janeiro, RJ, Brazil) and o-phenylenediamine (Sigma) were added to the wells and incubated for $5 \mathrm{~min}$ in the dark. The reaction was stopped with blocking solution (4 $\mathrm{N} \mathrm{H}_{2} \mathrm{SO}_{4}$ ), and the optical density (OD) readings were done at $492 \mathrm{~nm}$, using a microplate reader (SLT-SpectraSLT-Labinstruments, Salzburg, Austria).

As shown in Fig. 1A, the best results were obtained on wells coated with peptides P1, P2, P3, P6, and P7 and tested against the pool of positive serum samples using Costar 3590 flat-bottom polystyrene plate (Corning Incorporated Corning, New York, NY, US). The signals increased considerably $(p<0.0003)$ when the microplates were sensitized with the peptides P1, P2, P3, and P6 at $37^{\circ} \mathrm{C}$ for $30 \mathrm{~min}$ prior to overnight incubation at $4^{\circ} \mathrm{C}$ (alternative methodology). In contrast, the well coated with P7 presented statistically significant lower signal when compared with conventional methodology $(p=0.0019)$ (Fig. 1B). These results showed that the sensitization with peptides is an important step to improve the analytical sensitivity of ELISA test.

The peptides P1, P2, and P3 were produced based on the amino acid sequences of the peptides IMT164, IMT178, and IMT 180, described by Noya et al. (2001), who synthesized, under the t-boc manual procedure 17 peptides, comprising the entire Sm31 molecule. These authors evaluated the immunogenicity of these peptides in rabbits, obtaining polyclonal anti-peptide antibodies that were than tested for their ability to recognize Sm31 molecule in whole parasite extracts.

Although the relatively low (-1.411 to 0.333$)$ hydrophilic algorithms determined protein translated by ORF phase of Valli's ET03 clone have not suggested good antigenic indices for it, two peptides (P6 and P7) were synthesized for evaluating as antigenic substrate in our study, due to previous observations related to its high and consistent immunoreactivity against human anti-S. mansoni antibodies, as shown by Valli (2001). These peptides (P6 and P7) screening by ELISA, on Costar 3590 micro plates, showed strong immunoreactivity against positive control sera, with no reactivity against negative pool (Fig. 1A, B).

Peptides P4 and P5 lacked reactivity when tested against positive control sera pool, although the flexibility and hydrophilicity data have suggested good antigenic indices (data not shown). The low reactivity of the peptide P4 was not expected because SmHSP70 protein showed to be an important immunodominant target for humoral and cellular response in $S$. mansoni infection (Kanamura et al. 2002). It is conceivable that the antigenicity of the SmHSP70 requires other protein regions besides the $\mathrm{P} 4$ peptide. 

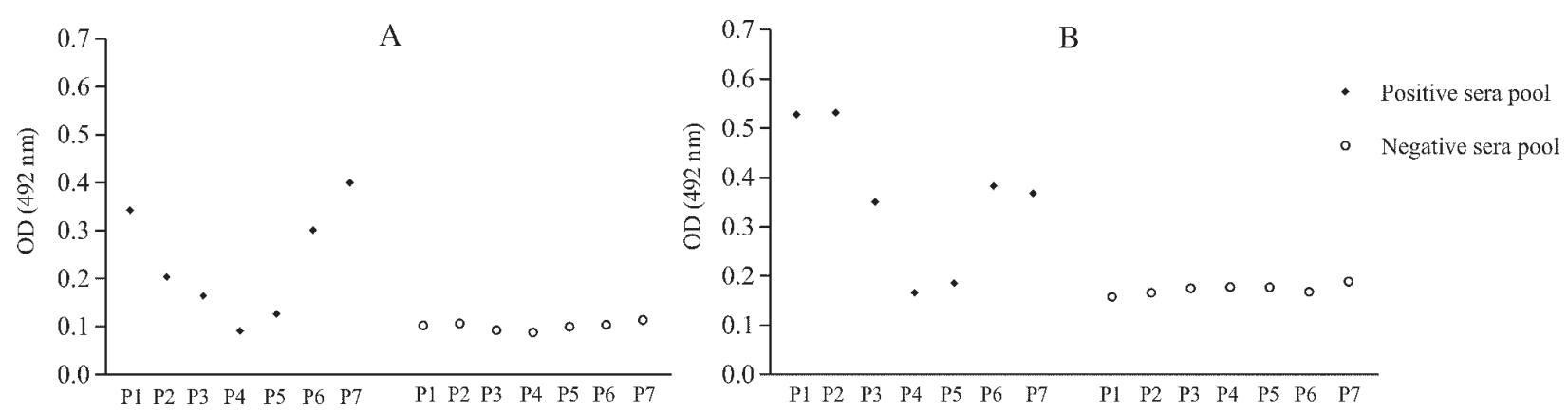

Fig. 1: peptides of Schistosoma mansoni tested by ELISA against S. mansoni positive and negative sera pools. A: conventional methodology; B: alternative methodology [Optical density (OD): mean of two experiments].

Further studies, involving other portions and epitopes of this protein, will be important to determine and synthesize new peptides with potential diagnostic value for schistosomiasis.

The low reactivity of the peptide P5 may be due the absence of polysaccharide moiety of the molecule. It has been shown that polysaccharides play an important role in determining the secondary structure of the antigen and therefore may be essential for immunoreactivity of molecules such as CCA.

In conclusion, peptides from catepsin B (P1, P2, P3) and ET03 clone (P6 and P7) seem to have high antigenicity when used as antigen on ELISA test for $S$. mansoni infection and may be useful for improvement immunodiagnosis of schistosomiasis.

\section{REFERENCES}

Arora SK, Melby PC, Sehgal S 1995. Lack of serological specificity of recombinant heat shock protein of Leishmania donovani. Cell Biol 73: 446-451.

Deelder AM, Kornelis D, Van Marck EAE, Eveleigh PC, Van Egmond JG 1980. Schistosoma mansoni: characterization of two circulating polysaccharide antigens and the immunological response to these antigens in mouse, hamster, and human infections. Exp Parasitol 50: 16-32.

Deelder AM, Van Dalen DP, Van Egmond JG 1978. Schistosoma mansoni: microfluoromethic determination of circulating anodic antigen and antigen-antibody complexes in infected hamster serum. Exp Parasitol 44: 216-224.

El-Sayed LH, Ghoneim H, Demian SR, El-Sayed MH, Tawfik NM, Sakr I, Abou-Basha LM, Renganathan E, Klinkert MQ, Abou-Rawash N 1998. Diagnostic significance of Schistosoma mansoni proteins Sm 31 and Sm32 in human schistosomiasis in an endemic area in Egypt. Trop Med Int Health 3: 721-727.

Expasy Proteomics Server (expert protein analysis system), http://au.expasy.org/cgi-bin/protscale.pl, access in: May 22, 2002.

Hopp TP, Woods KR 1983. A computer program for predicting protein antigenic determinants. Mol Immunol 20: 483-489.

Kanamura HY, Hancock K, Rodrigues V, Damian RT 2002. Schistosoma mansoni heat shock protein 70 elicits an early humoral immune response in $S$. mansoni infected baboons. Mem Inst Oswaldo Cruz 97: 1-6.

Klinkert MQ, Bommert K, Moser D, Felleisen R, Link G, Doumbo O, Beck E 1991. Immunological analysis of cloned Schistosoma mansoni antigens Sm31 and Sm32 with sera of schistosomiasis patients. Trop Med Parasitol 42: 319-324.

Moseley P 2000. Stress proteins and the immune response. Immunopharmacology 48: 299-302.

Moser D, Doumbo O, Klinkert MQ 1990. The humoral response to heat shock protein 70 in human and murine schistosomiasis mansoni. Parasite Immunol 12: 341-352.

National Library of Medicine. National Center for Biotechnology Information. GenBank: Sequence. Disponível em: http:/ /www.ncbi.nlm. nih.gov/Genbank/index.html. access in: May 22, 2002.

Noya O, De Noya AB, Ballen D, Bermúdez H, Bout D, Hoebeke J 2001. Immunogenicity of synthetic peptides from the Sm31 antigen (cathepsin B) of the Schistosoma mansoni adult. Parasite Immunol 23: 567-573.

Oliveira EJ, Kanamura HY, Lima DMC 2005. Efficacy of an enzyme-linked immunosorbent assay as a diagnostic tool for schistosomiasis mansoni in individuals with low worm burden. Mem Inst Oswaldo Cruz 100: 421-425.

Tort J, Brindley PJ, Knox F, Wolfe KH, Dalton JP 1999. Proteinases and associated genes of parasitic helminths. $A d v$ Parasitol 43: 161-166.

Valli LCP 2001. Imuno-seleção de Clones de Genoteca cDNA de Schistosoma mansoni Através de Anticorpos Dissociados de Imunocomplexos, $\mathrm{PhD}$ Thesis, Universidade de São Paulo, São Paulo, 107 pp.

Van Dam GJ, Seino J, Rotmans JP, Daha MR, Deelder AM 1993. Schistosoma mansoni circulating anodic antigen but not circulating cathodic antigen interacts with complement component C1q. Eur J Immunol 23: 2807-2812. 
